\title{
Molecular basis of aggressive disease in chronic lymphocytic leukemia patients with 11q deletion and trisomy 12 chromosomal abnormalities
}

\author{
AMIT K. MITTAL ${ }^{1}$, GANAPATI V. HEGDE ${ }^{1}$, PATRICIA AOUN ${ }^{4}$, ROBERT G. BOCIEK ${ }^{2}$, BHAVANA J. DAVE ${ }^{3}$, \\ AVADHUT D. JOSHI ${ }^{1}$, WARREN G. SANGER ${ }^{3}$, DENNIS D. WEISENBURGER ${ }^{4}$ and SHANTARAM S. JOSHI ${ }^{1}$ \\ Departments of ${ }^{1}$ Genetics, Cell Biology and Anatomy, ${ }^{2}$ Internal Medicine, ${ }^{3}$ Munroe-Meyer Institute for Genetics \\ and Rehabilitation, ${ }^{4}$ Department of Pathology and Microbiology, University of Nebraska Medical Center, \\ Omaha, NE 68198-6395, USA
}

Received January 25, 2007; Accepted February 27, 2007

\begin{abstract}
In B-cell chronic lymphocytic leukemia (CLL), Rai stage, immunoglobulin gene mutational status, chromosomal abnormalities, CD38 and ZAP-70 expression were used as prognostic markers. In this study, to understand the molecular basis of chromosomal abnormalities leading to tumor progression, 90 CLL patients were grouped into poor prognosis (with 11q deletion and trisomy 12) and good prognosis (with normal karyotype and $13 \mathrm{q}$ deletion) and their clinical outcome was assessed. Gene expression profiles of 35 CLL samples with poor outcome ( $11 \mathrm{q}$ deletion, $\mathrm{n}=9$; trisomy $12, n=5)$ and good outcome (13q deletion, $n=13$; normal karyotype, $n=8$ ) were analyzed using oligonucleotide microarray. Significance analysis of microarray (SAM) identified 27 differentially expressed genes between these two subgroups with significant overexpression of ATF5 and underexpression of CDC16, PCDH8, SLAM, MNDA and ATF2 in CLL patients with poor outcome. ATF5 gene expression in CLL was further studied because of its role in the regulation of cell cycle progression/differentiation and apoptosis. The overexpression of ATF5 was confirmed by real-time PCR using 39 CLL samples from the poor and good outcome groups. ATF5 was significantly $(\mathrm{p}<0.001)$ overexpressed in the poor outcome group. Furthermore, ATF5 expression was significantly higher in the 11q deletion as well as trisomy 12 group alone compared to the $13 q$ deletion and normal karyotype groups. ATF5 overexpression was also associated with significantly $(\mathrm{p}=0.04)$ shorter time to treatment. Similarly, expression of five underexpressed
\end{abstract}

Correspondence to: Professor Shantaram S. Joshi, Department of Genetics, Cell Biology and Anatomy, University of Nebraska Medical Center, 986395 Nebraska Medical Center, Omaha, NE 68198-6395, USA

E-mail: ssjoshi@unmc.edu

Key words: chromosomal abnormalities, chronic lymphocytic leukemia, 11q deletion, trisomy 12, ATF5 genes also correlated with longer time to treatment. Thus, this report demonstrates that ATF5 may be one of the key genes involved in increased proliferation and survival in $11 \mathrm{q}$ deletion or trisomy 12, whereas CD16, CD86, SLAM, MNDA and ATF2 may be involved in the decreased proliferation of CLL cells with $13 q$ deletion or normal karyotype.

\section{Introduction}

B-cell chronic lymphocytic leukemia (CLL) is a heterogeneous disease characterized by a highly variable clinical course. Some patients have an aggressive disease and they succumb within a short period after diagnosis, whereas others exhibit a more stable and indolent disease and survive for a prolonged period without any need of therapy $(1,2)$. To predict prognosis and to decide the treatment strategy after the diagnosis, prognostic factors are of immense importance (3). Based on tumor cell burden, two major clinical staging systems were developed by Rai et al (4) and Binet et al (5). Although these staging systems are useful, their ability to predict outcome, particularly in early stages of the disease, is limited (6). Therefore, prognostic markers related to the biology of CLL, such as chromosomal abnormalities and immunoglobulin variable heavy chain $\left(\mathrm{IgV}_{\mathrm{H}}\right)$ mutation status, are increasingly being evaluated for their prognostic value (7). Damle et al (8) and Hamblin et al (9) correlated the presence and absence of mutated $\operatorname{IgV}_{\mathrm{H}}$ with a favorable and an unfavorable clinical course, respectively. At present, $\operatorname{IgV}_{\mathrm{H}}$ mutation status is considered as one of the most reliable prognostic markers $(6,7)$. However, this analysis requires technically skilled DNA sequencing, which is also not commonly available for routine clinical practices. Thus, easily determined prognostic markers are required. In addition, to understand the molecular basis of prognostic markers, such as in the case of cytogenetic abnormalities, CD38 and ZAP70 expression may help identify genes to develop therapeutic strategies.

Chromosomal abnormalities are seen in $\sim 80 \%$ of CLL cases by fluorescence in situ hybridization (FISH) (10). We $(11,12)$ and others $(10)$ have shown that CLL cases with $11 \mathrm{q}$ 
deletion, 17p deletion and trisomy 12 have a more aggressive disease compared to CLL with $13 \mathrm{q}$ deletion or normal karyotype. Krober et al (13) have shown that the frequency of occurrence of genomic aberrations was equal in the mutated and unmutated $\mathrm{V}_{\mathrm{H}}$ subgroups, but chromosomal abnormalities associated with poor prognosis, such as $11 \mathrm{q}$ deletion and $17 \mathrm{p}$ deletion, were almost exclusively found in the $\mathrm{IgV}_{\mathrm{H}}$ unmutated subgroup, whereas favorable abnormalities such as $13 q$ deletion were associated with $\operatorname{IgV}_{\mathrm{H}}$ CLL-mutated subgroups. Though genomic abnormalities are of independent prognostic value in multivariant analysis, little is known about the underlying genetic and molecular mechanisms responsible for aggressive or indolent clinical behavior of CLL patients. Therefore, in the present study CLL patients were divided into two groups, one with the $11 \mathrm{q}$ deletion and trisomy 12 as the poor outcome group and another with the $13 \mathrm{q}$ deletion and a normal karyotype as the good outcome group. We determined the differential gene expression between these two subgroups, and identified differentially expressed genes were confirmed using real-time PCR and/or correlated with time to treatment as a measure of disease progression. Thus, these results provide a molecular basis of chromosome abnormalities in CLL patients.

\section{Materials and methods}

Patients. In this study, CLL patients without treatment or patients who were not treated for the prior 6 months were included. All patients were diagnosed with CLL based on clinical and laboratory evaluations. Peripheral blood samples were collected from 90 different CLL patients with informed consent using an Institutional Review Board (IRB) approved protocol. To determine the molecular basis of chromosomal abnormalities on tumor progression in CLL, cytogenetic analysis was performed on 90 patients using FISH. The distribution of chromosomal abnormalities in these patients was as follows: $11 \mathrm{q}$ deletion $(\mathrm{n}=13,14.4 \%)$, trisomy 12 $(n=17,18.8 \%), 13 q$ deletion $(n=28,31.1 \%), 17 p$ deletion $(n=6,6.6 \%)$, and normal karyotype $(n=26,28.8 \%)$. The other patient information, such as age, gender, status of other prognostic markers, clinical stage and time to treatment, were obtained and used for the determination of clinical characteristics and outcome.

Isolation and characterization of CLL cells. Mononuclear cells (MNCs) were isolated from heparinized whole blood from CLL patients using Accu-Prep ${ }^{\mathrm{TM}}$ lymphocyte separation medium by Ficoll-Hypaque density centrifugation method (Accurate Chemical and Scientific Corp., Westbury, NY,
USA) as described previously $(11,14)$. The immunophenotypes of the isolated MNCs were determined by flow cytometry with flurochrome (phycoerythrin/PE or fluoroscein isothiocyanate/FITC)-labeled antibodies specific for cell surface markers (11). The cells were stained using the following flurochrome-conjugated antibodies: CD3-FITC, CD5-PE, CD19-PE, CD38-PE (BD Pharmingen, San Jose, CA, USA) and a combination of conjugated antibodies: CD3FITC and CD38-PE; CD5-PE and CD19-FITC; CD19-FITC and CD38-PE (12). The percentages of cells, positive for each cell surface marker, were determined by Becton Dickinson FACStar plus Flow cytometer. All samples used in the present study had $>90 \% \mathrm{CD}^{+} / \mathrm{CD} 19^{+}$cells.

Cytogenetic analyses. The cytogenetic analyses of CLL cells were performed using fluorescent in situ hybridization (FISH) by the Human Genetics Department at the University of Nebraska Medical Center according to a previously described method (15) using the following probes: LSI 11q23-ATM DNA probe, CEP 12-trisomy 12 DNA probe, LSI 17p deletion p53 DNA probe and D13S25 13q14 DNA probe (Vysis Inc., Downers Grove, IL, USA).

DNA microarray analysis. Total RNA was extracted from CLL cells by TRIzol ${ }^{\mathrm{TM}}$ reagent (Invitrogen, Carlsbad, CA, USA) (12). The purity of RNA was determined by $1 \%$ Trisacetate-EDTA (TAE) agarose gel electrophoresis and quantitation was performed by spectrophotometry. The CLL RNAs were used for gene expression profiling using DNA microarray chip (MWG Biotech, Germany, Human 10K oligo set A) consisting of 50-mer oligonucleotide representing 10,000 different genes. The RNA from CLL samples and Stratagene $^{\mathrm{TM}}$ reference mRNA were reverse transcribed, then labeled with $\mathrm{Cy} 3$ or $\mathrm{Cy} 5$ fluorescence dyes and hybridized with array chip as described earlier $(11,12)$. The hybridized microarray slides were scanned and images were collected by using an Axon 4000B scanner (Axon Instruments, Union City, CA, USA). The ratios of fluorescence intensity for each spot/gene/element were obtained by using GenePix 6.0 software. All data files were collated and analyzed using $\mathrm{BRB}$ analysis tools.

Quantitative real-time PCR for ATF5. Expression levels of RNA transcripts were determined by real-time PCR using gene-specific primers as provided in Table I. cDNA was prepared from $5 \mu \mathrm{g}$ of total RNA from each CLL sample (11). Complementary DNAs were mixed with primers and FastStart SYBR-Green Master mix (Roche, Indianapolis, IN, USA), and real-time PCR was performed using the MyiQ ${ }^{\mathrm{TM}}$

Table I. List of primers used in real-time PCR analysis.

\begin{tabular}{lcccc}
\hline Gene name & GenBank accession no. & \multicolumn{1}{c}{ Temperature $\left({ }^{\circ} \mathrm{C}\right)$} & Product size $(\mathrm{bp})$ \\
\hline ATF5 & NM_012068 & $\begin{array}{l}\text { Forward 5'-CTCCTCCTTCTCCACCTCAA-3' } \\
\text { Reverse 5'-GCCGACTTGTTCTGGTCTCT-3' }\end{array}$ & 60 & 143 \\
& NM_000194 & Forward 5'-AGGGTGTTTATTCCTCATGGAC-3' & 60 & 103 \\
HPRT & Reverse 5'-GTAATCCAGCAGGTCAGCAAAG-3' & & \\
\hline
\end{tabular}


Table II. Patient characteristics.

\begin{tabular}{|c|c|c|c|}
\hline Characteristics & $\begin{array}{c}\text { 11q deletion } \\
\text { and trisomy } 12 \\
\text { and } 17 \mathrm{p} \\
\text { deletion group }\end{array}$ & $\begin{array}{l}\text { 13q deletion } \\
\text { and normal } \\
\text { karyotype } \\
\text { group }\end{array}$ & p-value \\
\hline \multicolumn{4}{|l|}{ Age } \\
\hline Median & 61 & 64 & 0.419 \\
\hline Mean & 61.18 & 63.41 & \\
\hline \multicolumn{4}{|l|}{ Gender } \\
\hline Male & $24(66.7 \%)$ & $26(48.1 \%)$ & 0.067 \\
\hline Female & $12(33.3 \%)$ & $28(51.9 \%)$ & \\
\hline \multicolumn{4}{|l|}{$\mathrm{sB} 2 \mathrm{M}(\mathrm{mg} / \mathrm{l})$} \\
\hline Median & 3.05 & 2.25 & 0.039 \\
\hline Mean & 3.44 & 2.58 & \\
\hline \multicolumn{4}{|l|}{ CD38 } \\
\hline Median & 32 & 18 & 0.008 \\
\hline Mean & 42 & 23 & \\
\hline Mutation status & & & NA \\
\hline Mutated & $3(15 \%)$ & $11(84.6 \%)$ & \\
\hline Unmutated & $17(85 \%)$ & $2(15.4 \%)$ & \\
\hline Rai stages & $\mathrm{n}=23$ & $\mathrm{n}=38$ & NA \\
\hline 0 & $6(26.1 \%)$ & $17(44.7 \%)$ & \\
\hline 1 & $3(13.0 \%)$ & $10(26.4 \%)$ & \\
\hline 2 & $4(17.4 \%)$ & $3(7.9 \%)$ & \\
\hline 3 & $4(17.4 \%)$ & $1(2.6 \%)$ & \\
\hline 4 & $6(26.1 \%)$ & $7(18.4 \%)$ & \\
\hline Lymphadenopathy & & & NA \\
\hline Present & $17(70.84 \%)$ & $4(23.53 \%)$ & \\
\hline Absent & $7(29.16 \%)$ & $13(76.47 \%)$ & \\
\hline
\end{tabular}

Single-Color real-time PCR detection system (Bio-Rad, Hercules, CA, USA). PCR conditions used were $30 \mathrm{sec}$ at $95^{\circ} \mathrm{C}, 45 \mathrm{sec}$ each at $60^{\circ} \mathrm{C}$ and at $72^{\circ} \mathrm{C}$. HPRT, a housekeeping gene, was used as a control.

Statistical analyses. CLL patients were grouped on the basis of chromosomal abnormalities. The time period from diagnosis to the first treatment was used as a parameter of disease progression or clinical outcome in CLL patients in the present study. The Kaplan-Meier method was used to determine the distribution of time to treatment among grouped patients. The log rank test was also used to assess the relationship between groups of different chromosomal abnormalities with time to treatment. Relative gene expression levels obtained from real-time PCR were used as a measure of expression of ATF5. The mean expression levels of ATF5 for each sample were arranged and then divided into groups by their median value. The log rank test was performed to compare the time to treatment between the above and below median groups. Similar statistical analyses were performed to determine the relationship between expression of CD16, PCDH8, SLAM, MNDA and ATF2 by comparing their transcription levels obtained from DNA microarray data to time to treatment. A p-value $\leq 0.05$ was considered statistically significant.

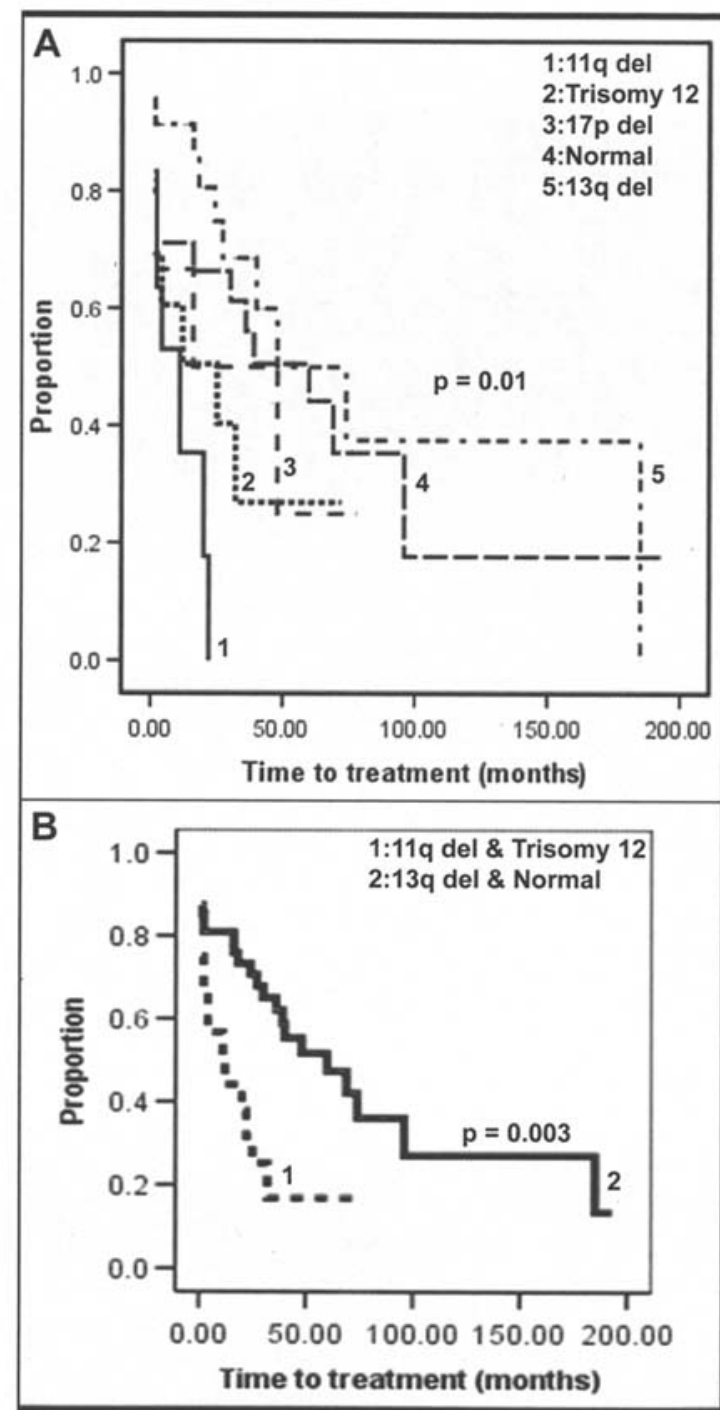

Figure 1. Association between chromosomal abnormalities and clinical outcome in CLL. (Panel A) Cytogenetic abnormality data in 90 CLL patients were used to determine the relationship between the cytogenetic abnormality and disease progression as measured by time to treatment using the log rank test. CLL patient subgroups with 11q deletion, trisomy 12 and $17 \mathrm{p}$ deletion were associated with significantly shorter time to treatment compared with CLL patients with $13 \mathrm{q}$ deletion and normal karyotype. (Panel B) CLL patients with 11q deletion and trisomy 12 cytogenetic abnormalities together and $13 \mathrm{q}$ deletion and normal karyotype cytogenetic abnormalities together were correlated with clinical outcome as measured by time to treatment using the log rank test. The poor outcome group was associated with significantly $(\mathrm{p}=0.003)$ shorter time to treatment compared with CLL patients from the good outcome group.

\section{Results}

Clinical evaluation. CLL patients with different chromosomal abnormalities were analyzed using different clinical parameters and the status of other prognostic markers. There was no statistically significant correlation between the age of the patients and the specific chromosomal abnormality subgroups. There was the indication of an $(p=0.067)$ association between gender and type of chromosomal abnormality where more male patients $(66.7 \%)$ had poor outcome chromosome abnormalities than female patients (33.3\%) (Table II). There was a significant association between CD38 expression in CLL cells and poor outcome chromosomal abnormality 


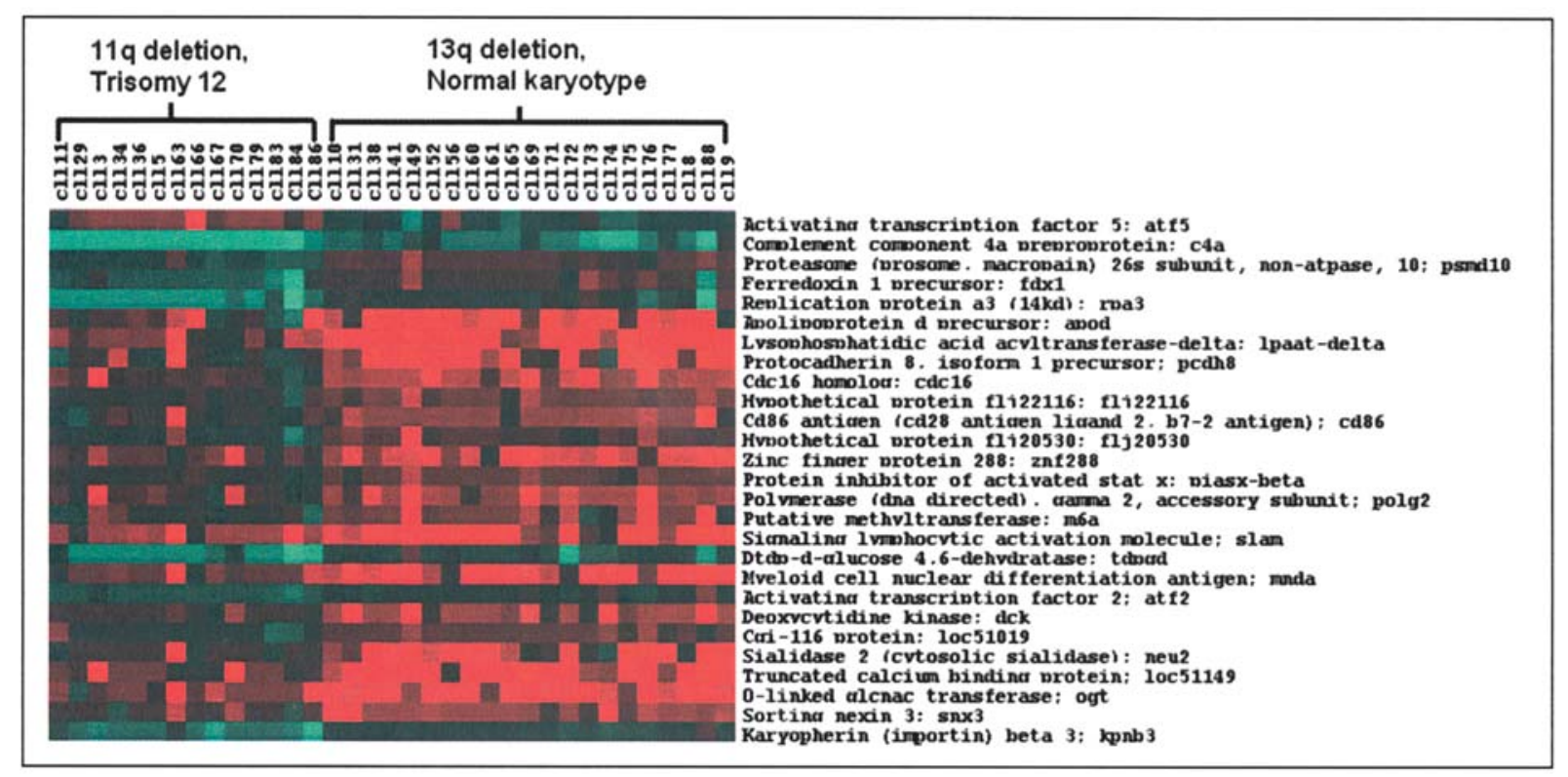

Figure 2. Supervised cluster analyses of genes. Supervised cluster analyses of differentially expressed genes in poor outcome chromosomal abnormality CLL subgroups with 11q deletion and trisomy 12 versus good prognosis CLL subgroups with $13 q$ deletion and normal karyotype. Significant analyses of microarray with a $90 \%$ confidence level were used.

( $\mathrm{p}=0.008)$, as well as between the presence of high levels of B-2 microglobulin in blood serum and poor outcome chromosomal abnormality, $(\mathrm{p}=0.039)$. In both of these cases, higher expression levels were associated with the poor outcome chromosomal abnormality group. CLL patients with $11 \mathrm{q}$ deletion and trisomy 12 poor outcome chromosome abnormalities were associated with unmutated $\operatorname{IgV}_{\mathrm{H}}$ and presence of bulky lymphadenopathy (Table II).

Cytogenetic analysis. Peripheral blood samples from ninety CLL patients were screened for known chromosomal abnormalities by FISH. The incidences/frequencies of each chromosomal abnormality were: $11 \mathrm{q}$ deletion $(\mathrm{n}=13) 14.4 \%$, trisomy $12(n=17) 18.8 \%, 13 q$ deletion $(n=28) 31.1 \%, 17 p$ deletion $(n=6) 6.6 \%$, and abnormalities and normal karyotype $(n=26) 28.8 \%$; which were consistent with the large studies performed earlier on CLL with chromosomal abnormalities $(10,11,16,17)$. In addition, as reported by our laboratory and others $(10,16,17)$, CLL patients with 11q deletion, trisomy 12 and $17 \mathrm{p}$ deletion were associated with a progressive disease. Furthermore, this report included about twice the number of patients compared to our earlier report. There was a significant association $(\mathrm{p}=0.01)$ between time to treatment and type of chromosomal abnormality (Fig. 1A). The 11q deletion and trisomy 12 patients were associated with shorter time to treatment (for 11q deletion, 11 months; for trisomy 12, 25 months) compared to $13 \mathrm{q}$ deletion and normal karyotype patients (for 13q deletion, 48 months; for normal karyotype, 60 months) at a $95 \%$ confidence level. Notably, unlike other reports, the $17 \mathrm{p}$ deletion chromosomal abnormality showed a similar pattern as trisomy 12 .

In the present study, to analyze the biological and clinical parameters among different abnormality groups, we used a mixed approach for hierarchical grouping for chromosomal abnormalities described by Dohner et al (10) and by us (11). In general, CLL patients with two or more chromosomal abnormalities are associated with poor outcome. We categorized the samples with both poor outcome chromosomal abnormalities (11q deletion or trisomy 12) and good outcome chromosomal abnormalities (13q deletion or normal karyotype). It is known that each chromosomal abnormality has independent prognostic value, and excluding one type of chromosomal abnormality will not affect the grouping of other abnormalities. The 11q deletion group, which had 11q deletions but not trisomy 12 , and the trisomy 12 group, which had trisomy 12 but not 11q deletions, were grouped together under the poor outcome group. The $13 \mathrm{q}$ deletion group, which contained neither 11q deletion nor trisomy 12 , and the normal karyotype group, where no chromosomal abnormality was detected were grouped together into the good outcome chromosomal abnormality group. When we analyzed these two groups together for clinical outcome, the poor outcome group had significantly $(\mathrm{p}=0.003)$ shorter time to treatment (12 months) compared to the good outcome group (60 months) (Fig. 1B). Thus these analyses confirmed the validity of our poor and good clinical outcome CLL subgroups. Subsequent analyses were based on these two groups.

Differential gene expression in cytogenetic abnormality CLL subgroups. CLL cells isolated from the poor outcome and the good outcome chromosomal abnormality CLL subgroups were analyzed for their gene expression pattern using oligonucleotide microarray technique. Significance analysis of microarray (SAM) was used to identify the differentially expressed genes. At the $99 \%$ and $95 \%$ confidence levels the number of differentially expressed genes between these two groups, were 4 and 7 respectively. At the low confidence level $(70 \%)$ two other genes (hypothetical protein flj22357 similar to epidermal growth factor and hypothetical protein flj12973) including ATF5 were overexpressed. In all these cases, ATF5 was the most significantly overexpressed gene in 


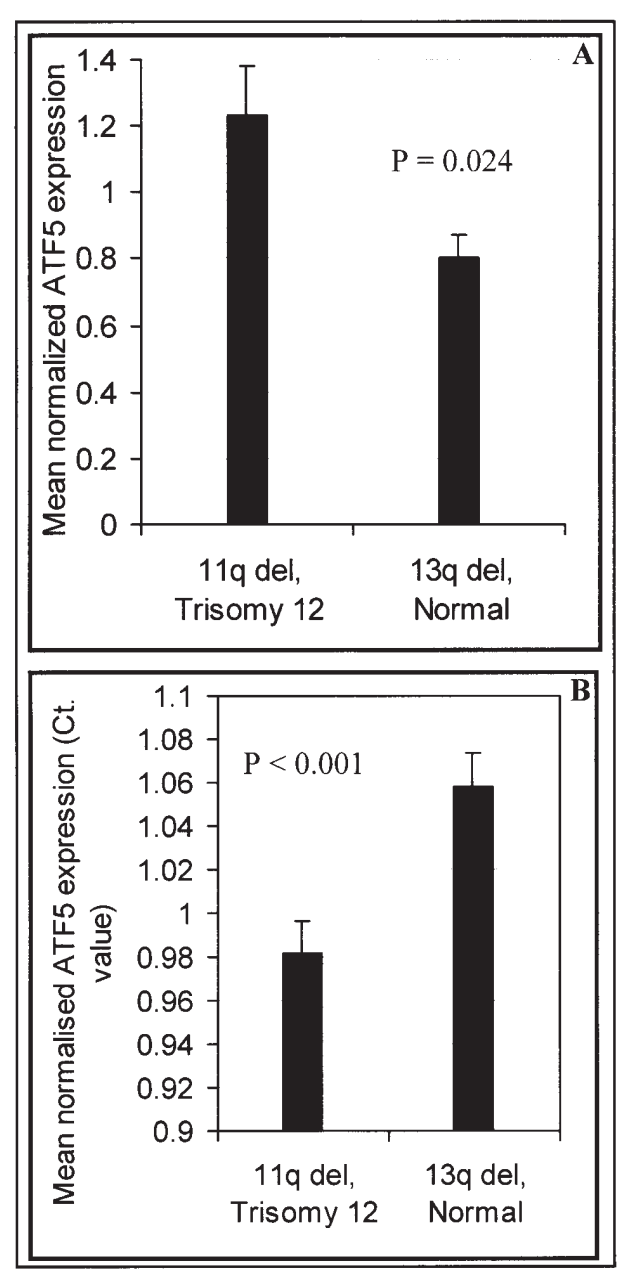

Figure 3. ATF5 expression in chromosomal abnormality CLL subgroups. (Panel A) Levels of ATF5 transcripts from the microarray data for CLL subgroups with $11 \mathrm{q}$ deletion and trisomy $12(\mathrm{n}=14)$ were compared to CLL subgroups with $13 \mathrm{q}$ deletion and normal karyotype $(\mathrm{n}=21)$. The statistical significance $(\mathrm{p}=0.024)$ between ATF5 expression between the two groups was determined with the t-test. (Panel B) ATF5 expression in chromosomal abnormality CLL groups using real-time PCR. The Ct value was determined for each CLL sample for their expression of ATF5, and the mean Ct value was calculated for each group. The $\mathrm{Ct}$ values were inversely proportionate $(\mathrm{p}<0.001)$ to ATF5 expression. Results from levels of transcripts as well as $\mathrm{Ct}$ values both indicated a higher expression of ATF5 in poor prognosis cytogenetic subgroups compared to good prognosis CLL subgroups.

the poor outcome CLL subgroup. ATF5 is a transcription factor involved in cell cycle regulation, cellular differentiation and apoptosis. Furthermore, when the analysis was performed at the $90 \%$ confidence level, the SAM analyses identified 27 consistently differentially expressed genes as detailed in Table III. Fig. 2 demonstrates a supervised cluster diagram showing expression levels of these 27 genes in red and green color. Among these genes, ATF5 was significantly $(\mathrm{p}=0.024)$ overexpressed in the poor prognosis chromosomal abnorm-ality subgroup compared to the good outcome subgroup; whereas CDC16, SLAM, MNDA, ATF-2 and PCDH8 were underexpressed in the poor outcome cytogenetic abnormality CLL subgroup. Although there were other genes that were underexpressed in the poor outcome CLL subgroups, only the genes involved in cell cycle regulation, proliferation and immune response were included in further analyses.

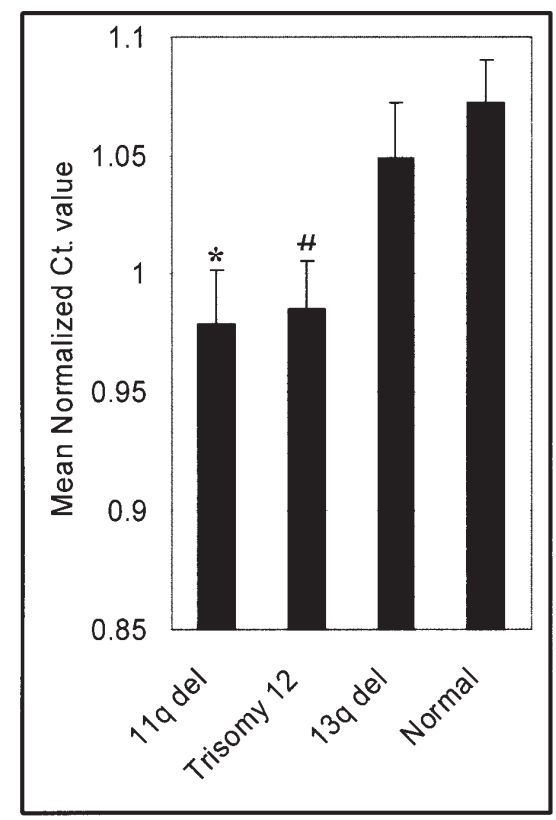

Figure 4. Linear relationship between ATF5 expression levels in CLL cells from different chromosomal abnormality subgroups. ATF5 expression in cytogenetic abnormality CLL subgroups as determined by real-time PCR showed a linearized pattern of $\mathrm{Ct}$ values. ATF5 was most significantly $\left({ }^{*} \mathrm{p}=0.0086\right)$ overexpressed in $11 \mathrm{q}$ deletion subgroups than trisomy 12 subgroup $\left({ }^{*} \mathrm{p}=0.0092\right)$ compared to $13 \mathrm{q}$ deletion and normal karyotype CLL subgroups. ATF5 expression levels (Ct values) demonstrated a linearized pattern with highest expression in 11q deletion subgroups and lowest in normal karyotype CLL subgroups.

Confirmation of overexpression of ATF5 in the poor outcome CLL subgroup. Since the SAM analyses identified ATF5 as one of the most significantly overexpressed genes in the poor outcome CLL subgroups, the overexpression was confirmed by real-time PCR. Fig. 3 shows the results of these expression value analyses. Fig. 3A shows the transcript levels from the microarray analyses from 35 different patient samples. There was a significant overexpression of ATF5 in the poor outcome chromosomal abnormality CLL subgroup compared to the good outcome chromosomal abnormality CLL subgroup. Since ATF5 is a key gene in the regulation of cell cycle and apoptosis, before embarking on in-depth studies on the role of ATF5 in CLL proliferation and survival, we elected to further confirm the ATF5 expression using real-time PCR analyses.

Fig. 3B shows the results of real-time PCR demonstrating the relative expression levels of ATF5 in the poor outcome chromosomal abnormality CLL subgroup as measured by $\mathrm{Ct}$ values compared to the good outcome CLL subgroup. Thus the real-time PCR analyses confirmed the overexpression of ATF5 corresponding to an aggressive disease. Fig. 4 shows a linear relationship between the expression levels of ATF5 in the CLL cells from different chromosomal abnormality subgroups in this study as determined by real-time PCR. These results demonstrated that ATF5 expression was highest in 11q deletion patients, second highest in trisomy 12 patients, third highest in $13 \mathrm{q}$ deletion patients and lowest in normal karyotype patients.

Correlation of ATF5 expression and time to treatment. Kaplan-Meier analysis was performed to determine whether 
Table III. Differentially expressed genes between poor and good prognosis chromosomal abnormality groups.

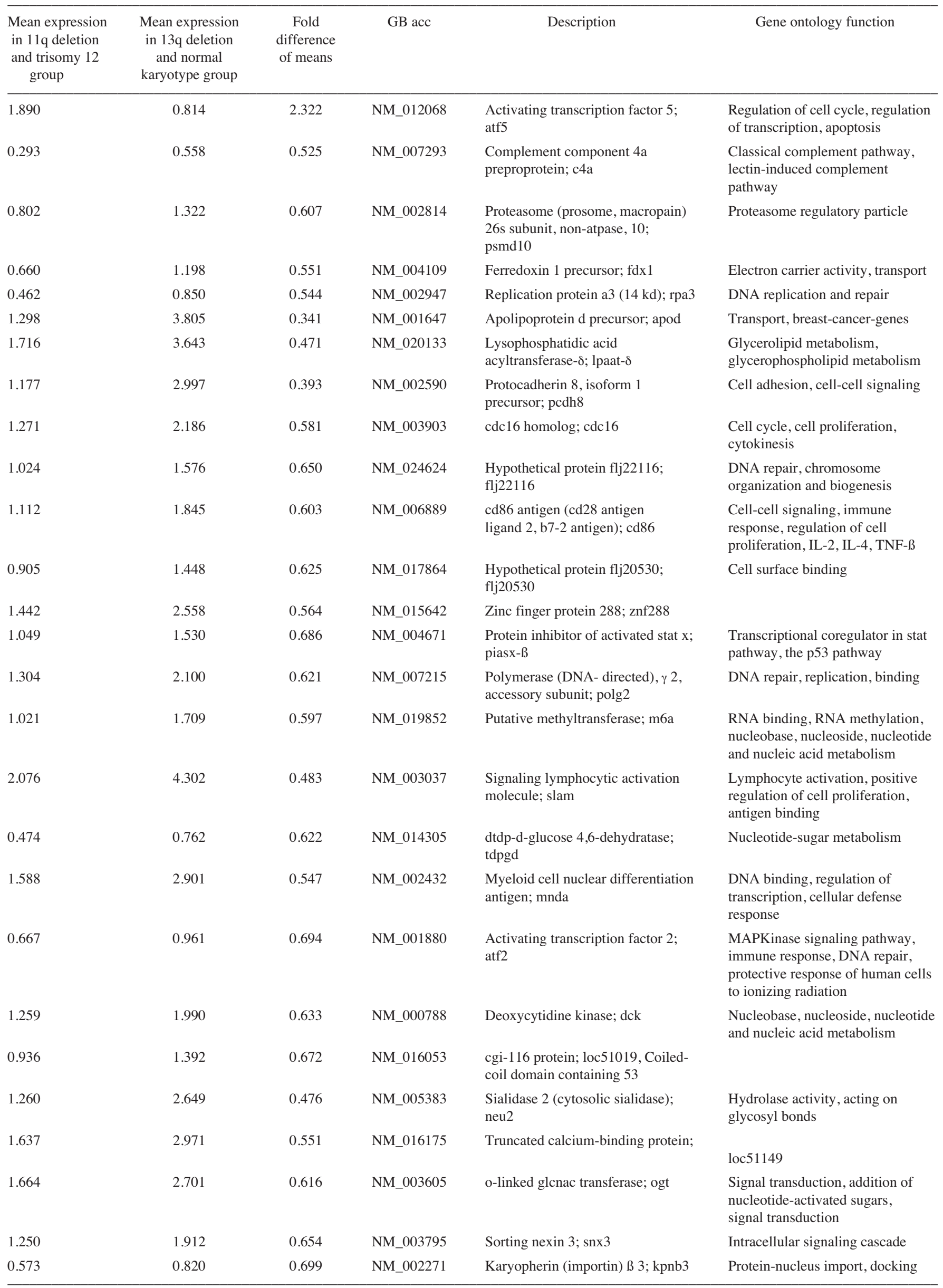




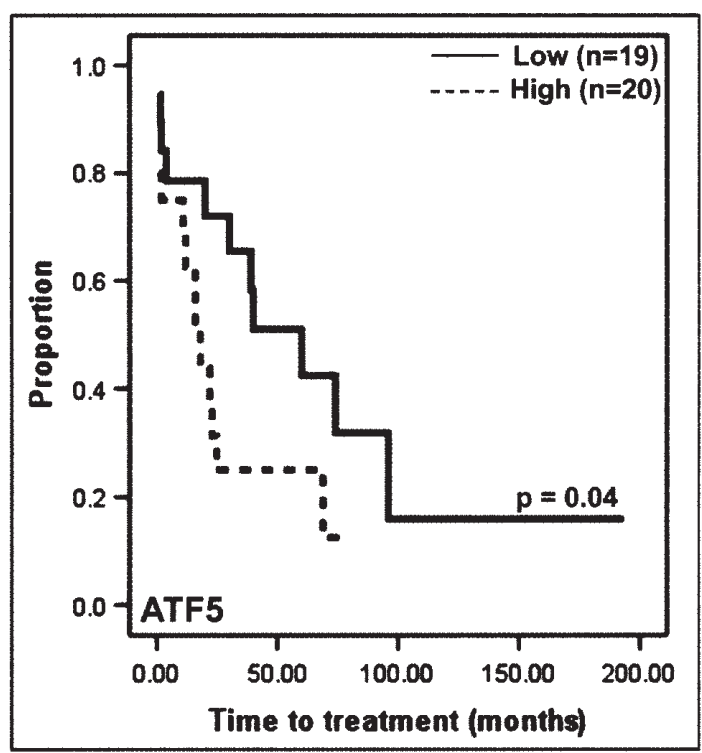

Figure 5. Association of ATF5 overexpression with disease progression. ATF5 expression levels were correlated with disease progression as determined by time to treatment. CLL patients with high expression of ATF5 had a significantly shorter time to treatment $(\mathrm{p}=0.04)$.

ATF5 expression was related to clinical outcome in CLL patients. In this analysis, ATF5 expression levels as measured by real-time PCR in different chromosomal abnormality CLL subgroups were correlated with time to first treatment using the log rank test. Fig. 5 demonstrates that CLL patients with high ATF5 expression had significantly shorter time to treatment $(\mathrm{p}=0.04)$ compared to CLL patients with low ATF5 expression. More specifically, CLL patients with high ATF5 expression had 16 months to first treatment compared to CLL patients with low ATF5 expression who had 60 months to first treatment.

Correlation of MNDA, ATF2, SLAM, CDC16 and PCDH8 with time to treatment. Genes such as MNDA, ATF2, SLAM, CDC16 and PCDH8 were underexpressed in the poor outcome chromosomal abnormality CLL group. In other words, these genes were overexpressed in the good outcome chromosomal abnormality CLL subgroup. In order to further elucidate the role of these genes in CLL cell biology and/or clinical behaviors, the expression levels of these genes were correlated with time to treatment in these CLL patients using the log rank test. Fig. 6 shows the results of these analyses. There was a significant correlation between the expression of MNDA, ATF2, SLAM, CDC16 and PCDH8 with time to treatment. The higher expression of MNDA was significantly associated with longer time to treatment ( 74 months) compared to CLL patients with low MNDA expression (24 months). Similarly, lower and higher expression of other genes was correlated with time to treatment: for ATF2, 22 vs 72 months; for SLAM, 24 vs 60 months; for CDC16, 20 vs 60 months and for PCDH8, 24 vs 60 months.

\section{Discussion}

This report describes the gene expression profiles associated with different chromosomal abnormality CLL subgroups.

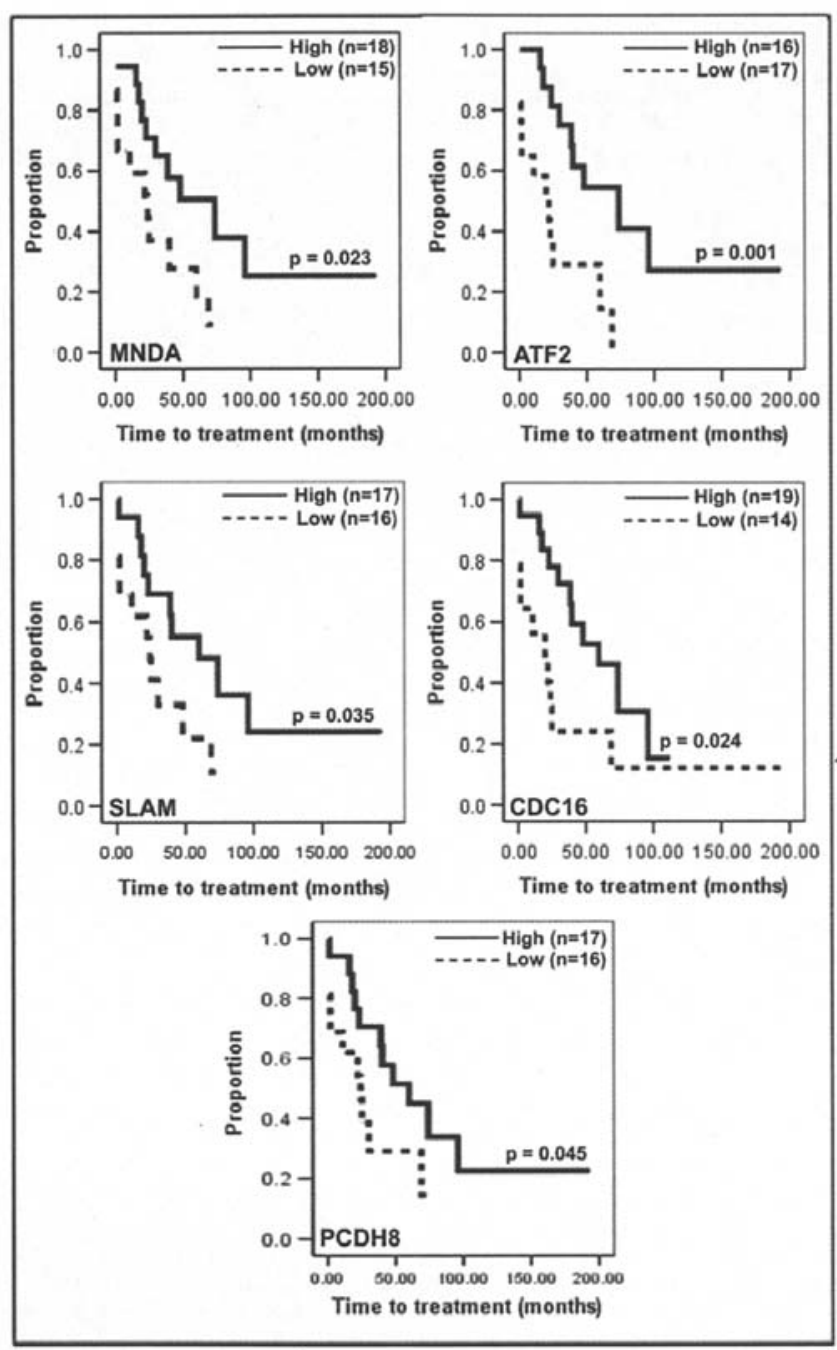

Figure 6. Association of underexpression of CD16, PCDH8, SLAM, MNDA and ATF2 with disease progression. The levels of transcripts for the underexpressed 5 genes were correlated with time to treatment using the log rank test. Underexpression of these genes was associated with significantly shorter time to treatment: MNDA, $\mathrm{p}=0.023$; CDC16, $\mathrm{p}=0.024$; SLAM, $\mathrm{p}=0.035 ; \mathrm{ATF} 2, \mathrm{p}=0.001$; and $\mathrm{PCDH} 8, \mathrm{p}=0.045$.

Cytogenetic analysis using FISH detected chromosomal abnormalities in $\sim 80 \%$ of CLL cases and the remaining $20 \%$ were of normal karyotype $(10,16-18)$. We and others have shown that chromosomal abnormalities influence the survival and progression of disease and, more specifically, 11q deletion, trisomy 12 and $17 \mathrm{p}$ deletion are associated with a more aggressive clinical outcome compared to $13 \mathrm{q}$ deletion and normal karyotype CLL subgroups. In previous studies $(11,12)$ we have shown that time to therapy was an endpoint to measure clinical progression in CLL patients. In accordance with previous studies $(10,11)$, our present study has shown that the $11 \mathrm{q}$ deletion and trisomy 12 group had the shortest time to treatment compared to the $13 \mathrm{q}$ deletion and normal karyotype CLL subgroups. Interestingly, disease progression in patients with $17 \mathrm{p}$ deletion fluctuated between trisomy 12 and $13 \mathrm{q}$ deletion. This could have been due to the small sample size, and it was hard to reach a conclusion. Though it is well established that $11 \mathrm{q}$ deletion, trisomy 12 and $17 \mathrm{p}$ deletion in CLL indicate poor prognostic values, the underlying molecular mechanism is still unknown. To follow 
up on our previous finding of a unique gene expression pattern in 11q deletion abnormality (11), in the present study we addressed the issue of differentially expressed key genes associated with poor prognostic chromosomal abnormality CLL patients with 11q deletion and trisomy 12 .

The gene expression profiles were compared between the poor outcome and the good outcome groups using oligonucleotide DNA microarray, and the SAM analyses revealed the differentially expressed genes ATF5, CDC16, PCDH8, SLAM, MNDA and ATF2. ATF5 was the most highly overexpressed gene in the poor outcome group. ATF5, also known as ATFx or ATF7, is a member of the activating transcriptional factor or cAMP response element-binding (ATF/CREB) family of basic leucine zipper (bZIP) protein $(19,20)$. Its role has been shown to regulate the differentiation process (19) and to inhibit apoptosis (21). Both differentiation and apoptosis play a major role in the cellular development cycle. Failure in one of these physiological processes may lead to cancer, where a cell either regains its ability to reenter the cell cycle and proliferate or escapes from natural death and begins accumulating in the body (21). Therefore, factors affecting these processes are very critical in cancer biology, and ATF5 is one of the factors which affects both processes. ATF5 overexpression has also been shown in malignant CLL cells compared to normal B-cells when activated with CD40 ligand (22). As it is well known that many cellular processes such as cell cycle, gene expression, protein synthesis and cellular transport are regulated through phosphorylation, ATF5 has also been identified as a factor which physically interacts with protein-tyrosine phosphatase (PTPase) (20).

We showed that overexpression of ATF5 was significantly associated with poor (11q deletion and trisomy 12) chromosomal abnormality CLL subgroups. CLL subgroups with $11 \mathrm{q}$ deletion were associated with the poorest outcome and had a significantly higher level of expression of ATF5 than the other chromosomal abnormality CLL subgroups. Trisomy 12 was second in predicting a grave outcome with second shortest time to treatment and second highest overexpression of ATF5. Our results not only confirmed the report of overexpression of the ATF5 gene in malignant CLL cells (22) but also demonstrated that relative expression of ATF5 was highly correlated with degree of malignancy, disease progression and outcome of patients. In addition, there was a linear relationship between the level of ATF5 expression and severity of the disease in CLL. Thus, our report is the first to show that higher ATF5 mRNA expression may be a marker of the aggressiveness of the peripheral blood CLL cells.

The majority of the underexpressed genes were related with nucleic acid replication, transcription, repair and metabolism (RPA3, POLG2, M6A, TDPGD, MNDA, ATF2, DCK), which indicated a tendency towards an insufficiency of functions to maintain the integrity of the genome in the poor outcome chromosomal abnormality group compared to the good outcome chromosomal abnormality group. In addition, genes related with immune response and cell adhesion or signaling or activation such as CDC16, CD86 antigen, SLAM, PCDH8, PIASX- $\beta$, MNDA, SNX3 were also underexpressed, which showed poor immunity and lymphocyte trafficking in the poor prognosis group. These results agree with the finding that decreased expression of genes related to cellular adhesion indicates low patient survival (23) or poor outcome. Moreover, ATF2 was underexpressed in the poor outcome chromosomal abnormality group. Interestingly, ATF2 is involved in multiple intracellular signal transduction pathways and is considered a candidate tumor suppressor gene, which plays a role in the development of human lung cancer, neuroblastoma and breast cancer (24).

In summary, these results identify the differentially expressed genes and demonstrate an association of overexpression of the ATF5 gene and underexpression of MNDA, ATF2, SLAM, CDC16 and PCDH8 with poor outcome chromosomal abnormality CLL patients. ATF5 may be one of the key genes responsible for inducing cell proliferation in CLL patients with 11q deletion and trisomy 12 chromosomal abnormalities, while other genes such as MNDA, ATF2, SLAM, CDC16 and PCDH8 may inhibit cell proliferation in CLL patients with $13 \mathrm{q}$ deletion and normal karyotype. Furthermore, these results open new avenues to further explore the details of the mechanisms of cytogenetic abnormality-induced disease progression.

\section{Acknowledgements}

This study was supported by CLL Foundation, Houston, TX. We wish to thank Ms Jennifer Mobly and Ms Ruth Olson for their help in preparing this manuscript.

\section{References}

1. Rozman $\mathrm{C}$ and Montserrat E: Chronic lymphocytic leukemia. $\mathrm{N}$ Engl J Med 1333: 1052-1057, 1995.

2. Zwiebel JA and Cheson BD: Chronic lymphocytic leukemia: staging and prognostic factors. Semin Oncol 25: 42-59, 1998.

3. Schroers R, Griesinger F, Trümper L, Haase D, Kulle B, Klein-Hitpass L, Sellmann L, Dührsen U and Dürig J: Combined analysis of ZAP-70 and CD38 expression as a predictor of disease progression in B-cell chronic lymphocytic leukemia. Leukemia 19: 750-758, 2005.

4. Rai KR, Sawitsky A, Cronkite EP, Chanana AD, Levy RN and Paternack BS: Clinical staging of chronic lymphocytic leukemia. Blood 46: 219-234, 1975.

5. Binet JL, Auquier A, Dighiero G, et al: A new prognostic classification of chronic lymphocytic leukemia derived from a multivariate survival analysis. Cancer 48: 198-206, 1981.

6. Keating MJ, Chiorazzi N, Messmer B, Damle RN, Allen SL, Rai KR, et al: Biology and Treatment of Chronic Lymphocytic Leukemia. ASH Educational Book (The American Society of Hematology), pp153-175, 2003.

7. Shanafelt TD, Geyer SM and Kay NE: Prognosis at diagnosis: integrating molecular biologic insights into clinical practice for patients with CLL. Blood 103: 1202-1210, 2004.

8. Hamblin TJ, Davis Z, Gardiner A, Oscier DG and Stevenson FK: Unmutated $\operatorname{IgV}(\mathrm{H})$ genes are associated with a more aggressive form of chronic lymphocytic leukemia. Blood 94: 1848-1854, 1999.

9. Damle RN, Wasil T, Fais F, Ghiotto F, Valetto A, Allen SL, et al: $\mathrm{IgV}$ gene mutation status and CD38 expression as novel prognostic indicators in chronic lymphocytic leukemia. Blood 94: 1840-1847, 1999.

10. Döhner H, Stilgenbauer S, Benner A, et al: Genomic aberrations and survival in chronic lymphocytic leukemia. N Engl J Med 343: 1910-1918, 2000.

11. Dickinson JD, Smith LM, Sanger WG, Zhou G, Townley P, Lynch JC, Pavletic ZS, Bierman PJ and Joshi SS: Unique gene expression and clinical characteristics are associated with the 11 q23 deletion in chronic lymphocytic leukaemia. Br J Haematol 128: 460-471, 2005

12. Joshi AD, Dickinson JD, Hedge GV, Sanger WG, Armitage JO, Bierman PJ, Bociek RG, de Vetten MP, Vose JM and Joshi SS: Bulky lymphadenopathy with poor clinical outcome is associated with ATM down regulation in CLL patients irrespective of 11q23 deletion. Cancer Genet Cytogenet 172: 120-126, 2006. 
13. Krober A, Seiler T, Benner A, Bullinger L, Brückle E, Lichter P, Döhner H and Stilgenbauer S: VH mutation status, CD38 expression level, genomic aberrations, and survival in chronic lymphocytic leukemia. Blood 100: 1410-1416, 2002.

14. Wang P, Munger CM, Joshi AD, Pirruccello SJ and Joshi SS: Cytotoxicity of cord blood derived Her2/neu-specific cytotoxic T lymphocytes against human breast cancer in vitro and in vivo. Breast Cancer Res Treat 83: 15-23, 2004.

15. Aoun P, Blair HE, Smith LM, Dave BJ, Lynch J, Weisenburger DD, Pavletic ZS and Sanger WG: Fluorescence in situ hybridization detection of cytogenetic abnormalities in B-cell chronic lymphocytic leukemia/small lymphocytic lymphoma. Leuk Lymphoma 45: 1595-1603, 2004.

16. Dewald GW, Brockman SR, Paternoster SF, Bone ND, O'Fallon JR, Allmer C, James CD, Jelinek DF, Tschumper RC, Hanson CA, Pruthi RK, Witzig TE, Call TG and Kay NE: Chromosome anomalies detected by interphase fluorescence in situ hybridization: correlation with significant biological features of B-cell chronic lymphocytic leukaemia. Br J Haematol 121: 287-295, 2003.

17. Dohner H, Stilgenbauer S, Dohner K, Bentz M and Lichter P: Chromosome aberrations in B-cell chronic lymphocytic leukemia: reassessment based on molecular cytogenetic analysis. J Mol Med 77: 266-281, 1999.

18. Stilgenbauer S, Lichter P and Dohner H: Genetics of chronic lymphocytic leukemia: genomic aberrations and $\mathrm{VH}$ gene mutation status in pathogenesis and clinical course. Leukemia 16: 993-1007, 2002.
19. Angelastro JM, Ignatova TN, Kukekov VG, Steindler DA, Stengren GB, Mendelsohn C and Greene LA: Regulated expression of ATF5 is required for the progression of neural progenitor cells to neurons. J Neurosci 23: 4590-4600, 2003.

20. Peters CS, Liang X, Li S, Kannan S, Peng Y, Taub R and Diamond H: ATF-7, a novel bZIP protein, interacts with the PRL-1 protein-tyrosine phosphatase. J Biol Chem 276: 13718-13726, 2001.

21. Persengiev SP, Devireddy LR and Green MR: Inhibition of apoptosis by ATFx: a novel role of member of the ATF/CREB family of mammalian bZIP transcription factors. Genes Dev 16: 1806-1814, 2002.

22. Gricks CS, Zahrieh D, Zauls AJ, Gorgun G, Drandi D, Mauerer K, Neuberg D and Gribben JG: Differential regulation of gene expression following CD40 activation of leukemic compared to healthy B cells. Blood 104: 4002-4009, 2004.

23. Stratowa C, Loffler G, Lichter P, Stilgenbauer S, Haberl P, Schweifer N, et al: c-DNA microarray gene expression analysis of B-cell chronic lymphocytic leukemia proposes potential new prognostic markers involved in lymphocyte trafficking. Int $\mathbf{J}$ Cancer 91: 474-480, 2001.

24. Woo IS, Kohno T, Inoue K, Ishii S and Yokota J: Infrequent mutations of the activating transcription factor- 2 gene in human lung cancer, neuroblastoma and breast cancer. Int J Oncol 20: 527-531, 2002. 